TENDENZEN DER BEVOLKERUNGSENTWICKLUNG WEST-BERLINS UND IHRE PROBLEMATIK FUR DIE STADTENTWICKLUNG

\author{
Bruno Backé
}

IZVLEČEK

UDK $911.3 .312(430,1-43.1)=30$

\title{
ZNAČLNOSTI RAZVOJA PREBIVALSTVA V ZAHODNEM BERLINU IN PROBLEMI RAZVOJA MESTA
}

Specifičen razvoj Zahodnega Berlina ima za posledico neugodne gospodarske procese in s tem povezano nazadovanje števila prebivalstva. Visok delež tujega prebivalstva vpliva na transformacijo mestnega jedra.

UDC $911 \cdot 3 \cdot 312(430,1-43 \cdot 1)=30$

WEST BERLIN DEVELOPMENT CHARACTERISTICS UND ITS SETTLEMENT DEVELOPMENT PROBLEMS

The specific development of West Berlin caused inconvenient economic processes and connected with that population regression. A high rate of foreign inhabitants influences the city centre transformation.

Um Berlin ist es ruhig geworden: Die politische Ruhe reicht von der Stabilisierung und Entspannung der politischen Lage um Berlin bis hin zur wiedergefundenen inneren politischen Ruhe West-Berlins: gewalttätige Demonstrationen sind fast schon zur Rarităt geworden; auch die Aufregungen um die Hausbesetzungen haben sich gelegt. Derzeit bewegt die Berliner einzig der Weggang ihres christdemokratischen Bürgermeisters, Richard von Weizsäckers, der in Bonn zum Bundespräsidenten gewählt werden wird.

Trotz dieser nach aussen hin positiven Zeichen steht es - so behaupte ich - nicht zum besten um die langfristige Existenzsicherung der Teilstadt West-Berlin. Es ist bekannt, dass die Stadt seit dem Ende des 2. Weltkrieges mit schweren politisch-geographischen Hypotheken belastet ist. Gelang es bisher mehr oder minder erfolgreich, die daraus resultierenden wirtschaftlichen Probleme zu meistern, so kann dies fur die

* Dr., Univ. prof., Institut der Geographie der Universităt für Bildungswissenschaften, 9010 Klagenfurt, Universitatstrasse 65-67 
zumindest ebenso problematische Bevölkerungssituation nicht behauptet werden. Ich werde deshalb diese Thematik in meinem Vortrag aufgreifen und uber die gegenwärtig erkennbaren "Tendenzen der Bevölkerungsentwicklung West-Berlins und ihre Problematik für die Stadtentwicklung" reden.

1. Lassen Sie mich zuerst zwei historische Ereignisse erwähnen, die für die Existenzsicherung der Teilstadt West-Berlin von grundlegender Bedeutung sind:

(1) Im Jahre 1972 traten die Berlin-Regelung und die deutsch-deutschen Zusatzvereinbarungen in Kraft. Durch diese Verträge wurde der politische Statusquo festgeschrieben bzw. garantiert und somit die Lebensfähigkeit West-Berlins gefestigt.

(2) Das zweite Ereignis sollte nicht einen Zustand festschreiben, sondern eine Veränderung, nämlich die seit dem Jahre 1958 sinkende Bevölkerungszahl West-Berlins, untersuchen und Vorschläge unterbreiten wie die aus den Fugen geratende Bevölkerungsentwicklung stabilisiert werden könne. Dazu wurde im Berliner Abgeordnetenhaus eine Kommission eingesetzt.

Wir haben heute - mehr als 10 Jahre nach diesen denkwürdigen Ereignissen - Anlass genug, die Frage zu stellen, ob und inwieweit die politische Stabilisierung und Entspannung (nach aussen und nach innen) in Berlin auch zu einer Stabilisierung der wirtschaftlichen und bevölkerungsmässigen Entwicklung West-Berlins geführt haben.

Die Grundthese meiner Ausführungen lautet:

Gelingt es nicht, neben dem wirtschaftlichen Aspekt der Lebensfähigkeit gleichzeitig und autonom (d. h. nur auf die Bevölkerungsentwicklung bezogen) das Bevölkerungsproblem zu lösen, ist das deklarierte Kardinalziel der Stadtentwicklung West-Berlins, nămlich "die stetige, langfristige Existenzsicherung der Stadt in Freiheit" nicht zu erreichen.

2. Ich beginne mit einer Skizzierung der politischen Rahmenbedingungen für die Bevölkerungsentwicklung. West-Berlins und setze fort mit den Aspekten der wirtschaftlichen Entwicklung der Stadt in den vergangenen Siebzigerjahren, ehe ich mich der Bevölkerungsproblematik selbst zuwende.

2.1 Die politischen Rahmenbedingungen West-Berlins haben ihre Ursache in der bis heute - im Sinne eines Friedensvertrages - nicht gelösten deutschen Frage; sie bestehen in 
1. der Isolierung West-Berlins,

2. der Teilung Berlins,

3. dem Verlust der Funktion der deutschen Hauptstadt und

4. der militärischen Präsenz der Alliierten in Berlin.

- Zur Isolierung West-Berlins:

West-Berlin wurde von seinem Umland praktisch zur Gănze abgeschnurt, ebenso von seinem mitteldeutschen Hinterland; es wurde durch die Zonengliederung Deutschlands răumlich vom abrigen Bundesgebiet getrennt.

West-Berlin ist zwar weder ein konstitutiver Bestandteil der Bundesrepublik Deutschland, noch darf es von der Bundesrepublik Deutschland regiert werden, jedoch ist es der Bundesrepublik Deutschland zugehörig, und zwar hinsichtlich der Wirtschafts-, Finanz-, Rechts- und Sozialordnung sowie im Hinblick auf das politische und kulturelle Leben; diese Zugehörigkeit findet ihren Ausdruck in eben diesen Bindungen, die aufrechterhalten und entwickelt werden können.

Die räumliche Isolierung wird durch freie, von der UDSSR garantierte Zugänge uberwunden.

Die räumliche Isolierung hat dazu gefuhrt, dass die Stadt alleine ohne die Hilfe durch die Bundesrepublik Deutschland - nicht lebensfähig ist.

- Zur Teilung Berlins ist zu sagen, dass sie auf das Jahr 1948 (Währungsumstellung) zurückgeht und seit dem Bau der Mauer im Jahre 1961 praktisch vollkommen ist.

- Der Verlust der Hauptstadtfunktion wirkt sich bis heute nachhaltig in wirtschaftlicher Hinsicht aus; er konnte durch keinerlei Massnahmen auch nur im Ansatz kompensiert werden. SCHRODER beziffert diesen Verlust mit ca. 1/3 der Wirtschaftskraft; ein zweites Drittel ging durch Kriegszerstörungen und Demontagen im sekundären Sektor verloren. Obwohl nach wie vor oder neu 14 Bundesbehörden ihren Sitz in West-Berlin haben, handelt es sich bei diesen Institutionen um sogenannte nachgeordnete Behörden; gegenuber obersten staatlichen Organen bestehen alliierte Vorbehalte.

- Schliesslich bildet die militärische Prăsenz der drei Alliierten, sprich: der Schutzmächte, deren Rechte und Verantwortlichkeiten aus der Kriegs- und Nachkriegszeit unverändert gelten, eine wesentliche Voraussetzung fur den Bestand der Weststadt. 
Die aktuellen Konsequenzen der politischen Rahmenbedingungen für die Lage der Stadt stellen sich auf der Grundlage der genannten Abkommen wie folgt dar:

\section{Die Existenz West-Berlins wird garantiert}

2. West-Berlin ist alleine nicht lebensfăhig: es bedarf der laufenden Finanzhilfe durch den Bund

3. Durch Besuchsregelungen traten menschliche Erleichterungen ein, d. h. die Folgen der Teilung konnten in menschlicher Hinsicht gemildert werden

4. Der akute Druck der politischen Unsicherheit (von aussen) konnte gemildert und die Konfrontation auf ein Minimum reduziert werden.

Im Prinzip wäre also zu erwarten, dass sich zumindest die wirtschaftlichen Bedingungen seit dem Jahre $1972 \mathrm{zu}$ konsolidieren und positiver als vorher $\mathrm{zu}$ entwickeln begannen.

2.2 Zur Skizzierung der wirtschaftlichen Rahmenbedingungen West-Berlins greife ich drei Bereiche heraus, die geeignet erscheinen, nicht nur die wirtschaftliche Lage und Entwicklung schlechthin zu beschreiben, sondern zugleich auch ihre Implikationen für die Bevölkerungsentwicklung erkennen zu lassen; es sind dies:

1. Die Lebensfähigkeit der Stadt,

2. die Entwicklung der Erwerbstätigen und

3. die Situation des verarbeitenden Gewerbes.

- Die Lebensfăhigkeit der Stadt wird in wirtschaftlicher Hinsicht auf gesetzlicher Grundlage durch das Berlinförderungsgesetz seitens der Bundesrepublik Deutschland garantiert und durch Transferzahlungen gesichert. Auf diese Weise alimentiert die Bundesrepublik Deutschland den West-Berliner Haushalt jăhrlich zu ca. $55 \%$; im Jahre 1983 waren dies 10,5 Mrd. DM. Dieser Betrag liegt in der Grössenordnung des jăhrlichen österreichischen Budgetdefizits. Es soll damit vor allem erreicht werden, dass West-Berlin mit dem Wachstum der Bundesrepublik Deutschland Schritt halten kann. Dieses $\mathrm{Ziel}$ ist nur teilweise erreicht worden: Im Zeitraum von 1970 bis 1980 blieb der Zuwachs des West-Berliner Bruttoinlandproduktes um durchschnittlich zwei Prozentpunkte, das ist ein Drittel des Gesamtzuwachses hinter jenem der Bundesrepublik Deutschland zurück.

- Die Entwicklung der Anzahl der Erwerbstätigen insgesamt im Zeitraum von 1970 bis 1983 zeigt an, dass die wirtschaftliche Bedeut- 
ung der Stadt abnimmt. Von 1970 bis 1979 nahm die Anzahl der Erwerbstätigen von 945.000 auf 835.000 , das sind $12 \%$, ab. Wăhrend in den meisten Städten und Grossstădten der Bundesrepublik Deutschland in den Siebzigerjahren Wirtschaftssparten wie Verkehrsund Nachrichtenwesen, Kreditinstitute und Versicherungswesen oder Handel mächtig wuchsen, stagnierten sie in West-Berlin oder erlebten sogar einen măchtigen Einbruch (Handel: - $22 \%$ ); der Bereich Dienstleistungen wuchs gering $(+4 \%)$, der Bereich Staat stărker (+ $12 \%)$; am stärksten ging die Beschäftigung im verarbeitenden Gewerbe (- $32 \%)$ und im Baugewerbe (- $24 \%)$ zurlick.

- Die Lage im verarbeitenden Gewerbe ist besorgniserregend, zumal dieses nach dem Verlust der Hauptstadtfunktionen die wichtigste Basis der fernbedarfstätigen Wirtschaftssparten bildet: im Zeitraum 1970 bis 1979 betrug der Ruckgang rund 100.000 Beschăftige, das sind $32 \%$ der Ausgangszahl (324.000).

Besonders krass ist der Verlust der Arbeitsplätze in der Industrie (Betriebe mit 20 und mehr Beschäftigten): 1970:265.000, Ende $1982: 163.000$, das sind $-38,5 \%$ in 12 Jahren. Im Vergleich mit der Bundesrepublik Deutschland (1965 - 1981 : - $11 \%)$ betrug der Verlust in Berlin-West im selben Zeitraum rund - $40 \%$. BerlinWest musste vor kurzem seinen ersten Rang als Industriestandort an Müchen abgeben.

Die Gründe für diesen Ruckgang sind nur zu einem kleineren Anteil in allgemein zu beobachtenden Trends, wie in der Wegrationalisier ung von Arbeitsplätzen zu suchen. Diese wurde in Berlin-West sogar noch durch die Berlin-Förderung (Investitionsförderung) wesentlich verstärkt. Daneben spielen Nachteile des Standortes WestBerlin eine wichtige Rolle: vor allem die stärkere Reduzierung des Arbeitsplatz-Angebotes multiregionaler westdeutscher Unternehmen in West-Berlin im Vergleich zur Bundesrepublik Deutschland; der Zusammenbruch der Kleiderindustrie; die Verlegung von Teilproduktionen Management- und Entwicklungsabteilungen nach Westdeutschland und das unterdurchschnittliche Wachstum von An-sich-Wachstumsbranchen in Berlin-West. Strukturell bedingt ist hingegen der starke Ruckgang kleinerer Betriebe infolge Uberalterungstendenz der Betriebsinhaber.

An dieser Stelle wird die enge Verkoppelung der Wirtschafts - mit der Bevölkerungsentwicklung, zumindest was die erwerbstătige Bevölkerung betrifft, deutlich. Trotz starker Gegensteuerung - zum Teil sogar gerade infolge dieser vermeintlichen Gegensteuerung durch Investitionsförderung - konnte durch den Staat der Ruckgang der Arbeitsplatze im verarbeitenden Gewerbe nicht eingebremst 
oder kompensiert werden, wie man sieht. Auf diese Weise sind negative Effekte der wirtschaftlichen Entwicklung auf die Bevölkerungsentwicklung unausbleiblich.

3. Ich komme damit zur Problematik der Bevölkerungsentwicklung. Lassen Sie mich die Bevölkerungssituation anhand einiger Befunde und ihrer Faktoren aufzeigen:

BEFUND 1: Die Entwicklung der Gesamtbevölkerung verläuft seit dem Nachkriegsbevőlkerungshöchststand am 1.1.1958 negativ (Abbildung 2): Die Bevölkerungsanzahl ging vom 1.1.1958 bis 1.1.1981 von 2,2 Mio. auf 1,9 Mio. zurück, das sind rund 330.000 Personen oder $15 \%$.

BEFUND 2: Das Vorliegen von drei amtlichen (!) Bevölkerungszahlen erschwert die Orientierung: Bezogen auf die Gesamtbevölkerung beträgt der maximale Unterschied zwischen den drei amtlichen Bevölkerungszahlen rund 283.000 Personen; dies entspricht in der Grössenordnung der Einwohnerzahl Ljubljanas. Es ist dies der Unterschied zwischen der Bevölkerungszahl der amtlichen, bundeseinheitlichen Bevölkerungsfortschreibung und der des Einwohnermelderegis. ters. In Abbildung 2 ist strichliert nur der kleinere Unterschied zwischen der amtlichen bundeseinheitlichen und der amtlichen West-Berliner Bevölkerungszahl (+102.000 Personen) eingetragen. Die Volkszăhlung, die hier alleine Aufklărung bringen könnte, ist schon seit dem Jahre 1970 überfällig.

BEFUND 3: Von diesen statistischen Unsicherheiten ist die sensibelste Grösse der Bevölkerungsentwicklung, nămlich die Anzahl der deutschen Bevölkerung, am stärksten betroffen, weil sie als Residualgrösse ermittelt, also nicht direkt fortgeschrieben wird (Gesamtbevölkerung minus ebenso unsichere nichtdeutsche Bevölkerung). Die Anzahl der deutschen Bevölkerung nimmt ungleich stärker ab als die Anzahl der Gesamtbevölkerung: im Zeitraum von 1961 bis 1981 betrug die Abnahme 517.000 Personen, das sind $24 \%$ !

BEFUND 4: Die natüliche Bevölkerungsentwicklung verlief und verläuft stark negativ (Abbildung 3 ). Eine starke Uberalterung der Bevölkerung (Abbildung 4) fuhrt bei sehr niedrigen Reproduktionsraten (Abbildung 5), die ihrerseits wieder bedingt sind durch die relativ geringe Besetzung reproduktionsfähiger Jahrgănge und die niedrige Fruchtbar- 
keit, zu hohen jahrlichen Geburtendefiziten (10 Personen/ 1.000 Einwohner/Jahr). Dieser bei der deutschen Bevölkerung ausgeprägte negative Effekt wird insgesamt durch gegenlăufige Komponenten der Auslănder (junge Bevölkerung/hohe Reproduktionsraten/relativ starke Besetzung reprăsentierfähiger Jahrgănge/hohe Fruchtbarkeit), schwach kompensiert. Die naturliche Bevölkerungsentwicklung beeinflusst die Gesamtentwicklung ausgeglichen negativ; der stark schwankende Verlauf der Gesamtkurve resultiert aus den Wanderungsbewegungen.

BEFUND 5: Die durch Wanderungen induzierte Bevölkerungsentwicklung verlief im Beobachtungszeitraum per Saldo völlig ausgeglichen ( \pm 11.000 Personen/Jahr); sie wird im wesentlichen durch zwei gegenläufige Wanderungskurven charakterisiert (Abbildung 3), deren Verlauf konjunkturabhăngig ist: Kurve 1 zeigt hohe Wanderungsgewinne mit dem Ausland (durchschnittlich 9.500 Personen/Jahr) bei starken Wanderungsverlusten Deutscher mit dem Bundesgebiet (Kurve 31) (durchschnittlich 14.700 Personen/Jahr). Die beiden übrigen Wanderungsbewegungen (Kurven 2 und $3_{2}$ ) fallen hingegen kaum ins Gewicht.

BEFUND 6: Bilanziert man die entsprechenden Salden fur den Beobachtungszeitraum von 1965 bis 1981 , so ergibt dies eine Bevölkerungsabnahme von rund 300.000 Personen in 16 Jahren, das sind $14 \%$.

Gravierend daran ist der Umstand, dass im Zuge dieser Verănderung die Anzahl der Deutschen 1.1.1965:2,166 Mio., 1.1.1981: 1,663 Mio. grob um rund 500.000 Personen (- $23 \%$ ) abnahm und die Anzahl der Auslănder $(1.1 .1965: 34.000,1.1 .1981: 233.000)$ um rund 200.000 Personen ( $+585 \%$ ) zunahm.

Dies leitet zu einem neuen Aspekt der Bevölkerungsproblematik, zur Ausländerproblematik uber; zuerst aber wollen wir noch den prognostischen Befund der Bevölkerungsentwicklung kennenlernen und Konsequenzen aus der bisherigen und künftigen Bevölkerungsentwicklung WestBerlins ableiten.

BEFUND 7: Die Prognose der Bevölkerungsentwicklung (Abbildung 7), die unter mehreren Annahmen errechnet wurde, zeigt auf, dass sich die Abnahme der deutschen Bevölkerung bzw. die Zunahme der ausländischen Bevölkerung sogar unter 
fast schon unrealistisch gtinstigen bzw. ungtinstigen Annahmen (Reduzierung der negativen Wanderungssalden der Deutschen um $1 / 3$ bzw. der positiven der Auslánder um 1/3) im gleichen drastischen Ausmass fortsetzen wird wie im Beobachtungszeitraum, und was die deutsche $\mathrm{Be}$ völkerung betrifft, sogar verstärkt gegenüber dem Zeitraum 1965 bis 1981 : statt $-1,8 \% / \mathrm{Jahr}:-2,1 \% / \mathrm{Jahr}$.

Der Deutlichkeit dieser Befunde dürfte nichts hinzuzufügen sein: sie zeigen in der Tat auf, dass die Bevölkerungsentwicklung als der kritische Faktor einer "stetigen langfristigen Existenzsicherung der Stadt" anzusehen ist.

Ein Anhalten dieser aufgezeigten Entwicklung birgt die Gefahr in sich, dass dieser Prozess von der deutschen Bevölkerung verstärkt wahrgenommen und möglicherweise als tendenzielles Aufgeben des Standortes West-Berlin betrachtet wird. Unter dieser Voraussetzung würde sich die Bevölkerungsabnahme Deutscher zu einem sich selbst tragenden, rasch voranschreitenden, kumulativen Schrumpfungsprozess ent-

- wickeln, der in der Folge nicht mehr durch wirtschaftliche Gegensteuerungsmassnahmen abgeschwächt, geschweige denn gestoppt werden könnte. Hinzu kommt die ohnehin stellenweise schon vorhandene Angst der deutschen Bevölkerung vor einer Uberfremdung durch die rasch anwachsende Anzahl der Ausländer. Die Inländer-Ausländerrelation dürte sich in den Achtzigerjahren von 12 auf 20 Ausländer je 100 Deutsche verschieben: dieser Durchschnittswert wird in kleineren răumlichen Einheiten schon heute weit übertroffen.

Fragt man nach den Steuerungsmöglichkeiten, so ist zu sagen: Der Abgang Deutscher infolge der natürlichen Bevölkerungsentwicklung ist unvermeidbar und hinzunehmen. Die mögliche Beeinflussung des Wanderungssaldos der deutschen Bevölkerung ist bei wesentlicher Verstärkung der derzeitigen Globalsteuerung sehr gering: nur $15 \%$ der Zuzugler bzw. der Fortzügler sprechen auf die derzeitigen Massnahmen überhaupt an. Und was die Steuerung der Ausländerentwicklung betriff, ist ausser einigen restriktiven Massnahmen hinsichtlich des Zuzuges keine Politik erkennbar.

\section{Befunde zur Ausländerproblematik}

Die Auslănderfrage bildete in West-Berlin zu keinem Zeitpunkt in die ubrige Bevölkerungsproblematik und -politik integrierten Gegenstand; sie wuchs vielmehr gegen Ende der Siebzigerjahre ziemlich rasch zu einem selbstăndigen, sicher|unlösbaren Problem heran. Der Zuzug von Auslăndern war lange Zeit hindurch erwünscht, 
(1) um die infolge des wirtschaftlichen Aufschwunges bei gleichzeitiger verstärkter Abwanderung Deutscher vermehrten offenen Stellen besetzen zu können (was verstărkt für unqualifizierte Tătigkeiten zutraf) und

(2) um die Bevölkerungskurve nicht zu stark absinken zu lassen.

Die Ausländerproblematik lässt sich anhand der folgenden Befunde verdeutlichen:

BEFUND 1: West-Berlin ist unter den westdeutschen Grossstădten mit mehr als einer halben Million Einwohnern nicht jene mit dem höchsten Auslănderanteil: es ist aber jene mit der bei weitem höchsten absoluten Anzahl der Ausländer und den höchsten Zunahmeraten (12\%, d.s. 233.000 Personen bzw. plus $33 \%$ seit 1974 ; hingegen: Frankfurt a. M.: $23 \%$, d.s. 126. 000 Personen bzw. plus $26 \%$ ).

BEFUND 2: Die Struktur der Ausländer nach Herkunftsgebieten ist sehr unausgeglichen: $49 \%$ oder 114.000 sind Turken - diese Gruppe stellt etwa im Bundesgebiet mit rund $33 \%(1,546$ Mio.) den bei weitem höchsten Anteil, 32.000 oder $14 \%$ kommen aus Jugoslawien, daneben spielen Griechen und Italiener noch eine gewisse Rolle, bleiben in ihren Anteilen jedoch unter je $3 \%$.

BEFUND 3: Der răumliche Ausbreitungsprozess der Auslănder in den Siebzigerjahren (Abbildungen 8 und 10) ist dadurch gekennzeichnet, dass zunăchst neben Industriegebieten vor allem die Stadtteile des wilhelminischen Wohngurtels entlang der Grenze zu Ost-Berlin aufgesucht werden:

Wedding, Tiergarten und Kreuzberg (Abbildung 1); es sind Gebiete mit schlechter Bausubstanz, hohem Anteil an Substandardwohnungen, hohem Arbeiteranteil, relativ hoher Bevölkerungsdichte sowie z.T. mit hohem Anteil an Wohnbevölkerung im Alter von 60 und mehr Jahren und z. T. mit hohem Frauenanteil und hohem Anteil an Stimmen der Alternativen Liste. Hingegen werden die Villengebiete und Gebiete mit lockerer Verbauung und hohem Sozialstatus im Sudwesten, Suden und Norden der Stadt sowie von der Bausubstanz her bessere Gebiete im wilhelminischen Wohnglirtel nebst Gebieten mit junger Bevölkerung (Satellitenstådte "Falkenhagener Feld", "Mărkisches Viertel", "Gropiusstadt") von Ausländern bis in die jüngste Zeit hinein gemieden. Wo 
herabgewohnte Gebiete unmittelbar an die West-Berliner $\mathrm{Ci}$ ty grenzen, dringen die Ausländer bereits in Citybereiche ein und verleihen dort den Problemen der Stadterneuerung einen besonderen Akzent (Abbildung 9).

BEFUND 4: Ein dramatischer Akzent ist fur bestimmte Gebiete mit sehr starker Verdichtungstendenz im Zuge des Ausbreitungsprozesses zu konstatieren: in manchen statistischen Gebieten erreichten die Ausländerquoten am 1.1.1981 Werte in der Grössenordnung von $25 \%$ und mehr.

Gründe:

+ stark gestiegene Zuzugsraten,

+ Familienzusammenführung,

+ hoher Zuwachs an Kindern und

+ starke Segregationstendenz (am 1.1.1981 = 35; Vergleich: Akademiker $=34 ;$ Wien am 31.12.1972: 12).

Fazit der Ausländerproblematik: Die hohe Zahl der Auslănder, ihre răumliche Konzentration, ihre kulturelle Distanz und ihre zunehmende Aufenthaltsdauer rufen bei der deutschen Bevölkerung Angst vor Uberfremdung hervor, zumal die Ausländer, insbesondere die Türken weder bereit noch in der Lage sind, den Erwartungen der deutschen Bevölkerung, sich an deren eigene Lebensgewohnheiten und Wertvorstellungen anzupassen, zu entsprechen. Der Zeitpunkt fur eine erfolgreiche Integrationspolitik scheint bereits verstrichen zu sein. Andere Lösungsmöglichkeiten sind nicht in Sicht.

Lassen Sie mich mit der folgenden Bemerkung schliessen: Angesichts eines so gewaltigen latenten Konflikpotentials in den Dimensionen Bevölkerungsstruktur und Bevölkerungsentwicklung kann abschliessend formuliert werden: Ist West-Berlin in der Lage, seine Bevölkerungsprobleme schrittweise zu lösen, so leistet diese Stadt selbst den vielleicht wesentlichsten Beitrag zu ihrer eigenen stetigen langfristigen Existenzsicherung in Freiheit. 


\section{ZNAČILNOSTI PREBIVALSTVENEGA RAZVOJA V ZAHODNEM BERLINU IN Z NJIM POVEZANI PROBLEMI MESTNEGA RAZVOJA}

Osnovni problemi Zahodnega Berlina so geopolitičnega značaja: njegova izolacija, delitev, izguba vloge nemške prestolnice ter pristojnost in prisotnost zaveznißkih okupacijskih sil. Ceprav je obstoj Zahodnega Berlina zagotovljen, mesto ni sposobno živeti brez pomoči Zvezne republike Nemčije. Gospodarski položaj pa vpliva na prebivalstveni razvoj v mestu, zaradi cesar prihaja do sprememb $v$ številu in strukturi prebivalcev Zahodnega Berlina.

Delež aktivnih prebivalcev stalno nazaduje, število zaposlenih seje od leta 1970 dalje zmanjšalo od 945.000 na 835.000 oseb. Nazadujejo pa tudi gospodarske dejavnosti, ki v drugih območjih ZR Nem ¿ije izkazujejo izrazito rast, trgovina $(-22 \%)$, proizvodna obrt (-32\%), gradbeništvo $(-24 \%)$. Še najbolj občutijo gospodarsko stagnacijo industrijska podjetja, ki so imela leta 1970 še 265 tisoð zaposlenih, v letu 1982 pa le še 163 tisoč $(-39 \%)$. Upad je za $40 \%$ večji kot v ZRN $(-11 \%)$.

Demografski razvoj kaže nazadovanje števila prebivalcev za petnajst odstotkov med leti 1958 in 1981 na okrog 1,900.000. Delež nemškega prebivalstva najhitreje upada $(-24 \%)$. Deloma je temu pripomogel negativni prirodni prirastek, povezan $\mathrm{z}$ ostarelo strukturo mestnega prebivalstva.

Migracijska gibanja so si veð ali manj izenačena, le strukture migrantov so različne. Medtem, ko se je v obravnavanem obdobju v ZRN odselilo pretežno nemško prebivalstvo ( 500.000 oseb), se v Zahodni Berlin intenzivno doseljujejo tujci, ki jih je bilo leta 1981 že 233.000 (+585\%). Delež tujcev je med največjimi v ZRN (12\%) in se najhitreje povečuje $(+33 \%)$. Najvec se je doselilo Turkov $(49 \%)$ in Jugoslovanov $(14 \%)$. Do zgostitve tujerodnega prebivalstva prihaja $v$ posameznih mestnih Cetrtih (npr. Wedding. Tiergarten, Kreuzberg). 


\section{ABBILDUNGEN}

\section{OBERSICHTSKARTCHEN: BERLW-WEST}

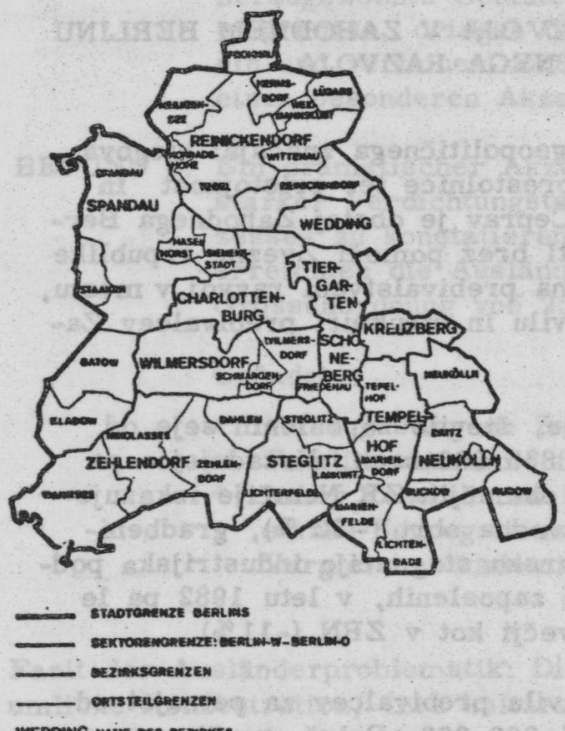

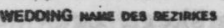

Leant me oes ortstencs

Abb. 1
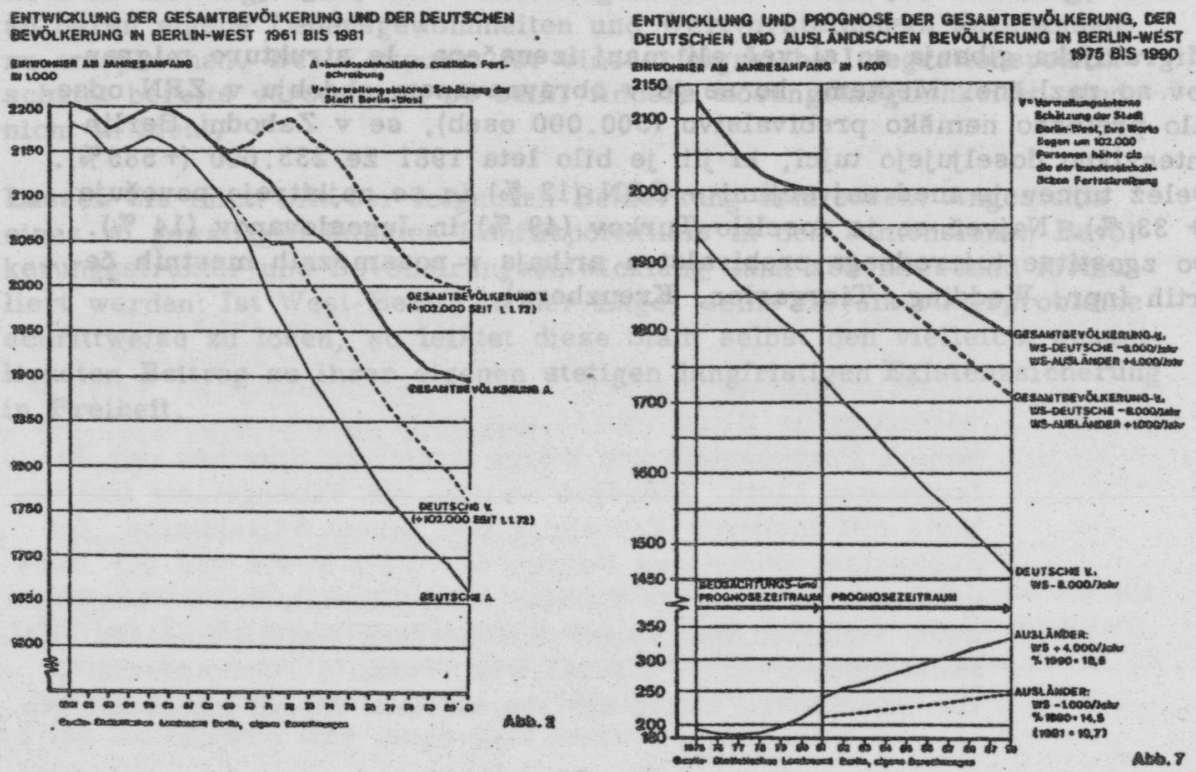


\section{AUSLANDERANTEILE IN DEN STATISTISCHEN GEBIETEN VON BERLIN-WEST}

AM 27.5. 1970

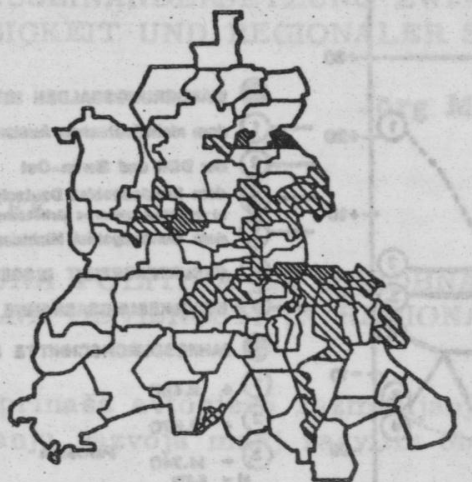

AN DER WOHWaEVUKERUNG

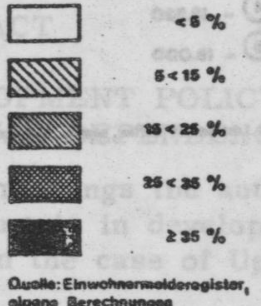

olgeno Berechnungea

STATISTISCHE CEBIETE MIT STARKER AUSPRĂGUNG DES FAKTORS
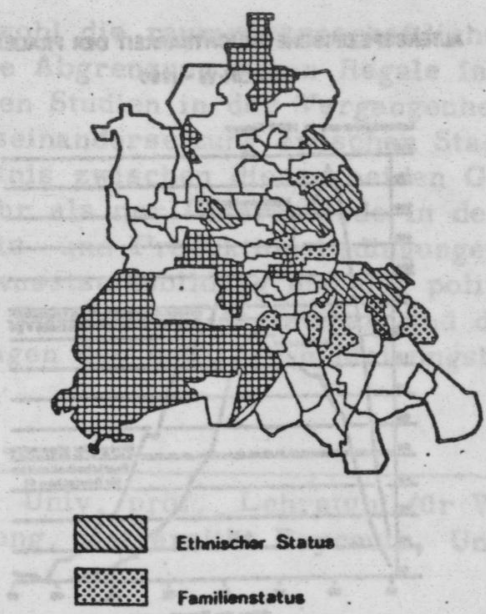

$\%$

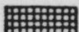

Ethnischer- und Familienstatue

Sozisler Status
AM LL 1981

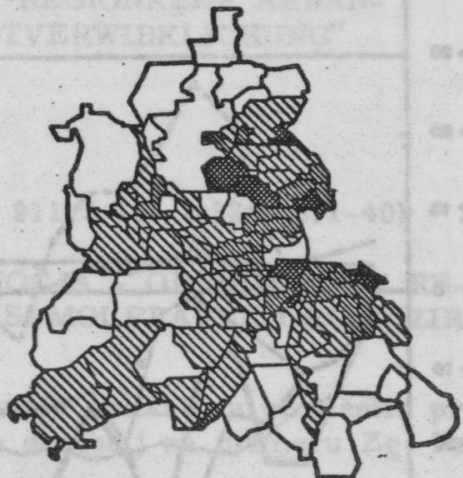

27. ล. ฆ70:

8. 2 เอย:

בxam

ves $\%$

บ\%

es\% $\%$

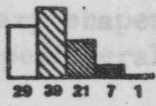

ate

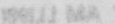
MACH STATISTISCHEN GEBIETEM YOM 27. 6.1970 an ZUA L.L.19al

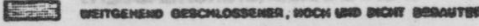

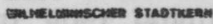

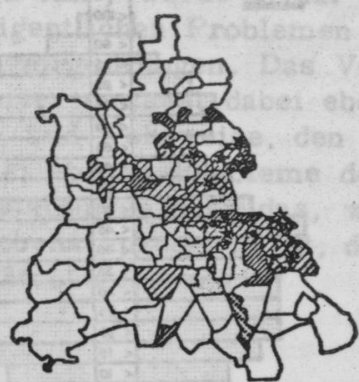

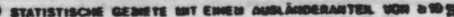

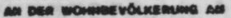

(8)

$$
\begin{array}{l|l|l}
x .5000 & 22000
\end{array} 21200
$$

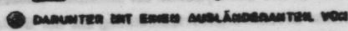

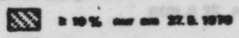

- $10 \%-212$

59

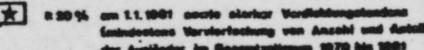

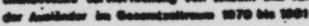
C 
BEVŎLKERUNGSENTWICKLWNG DER STADT BERLIN-WEST 1965 BIS 1980

DURCH GEBURTENDEFIZIT UNO AUSGEWÄHLTE WANDERUNGSSALDEN

DERSONEN W 1000

PERSONEN W 1000

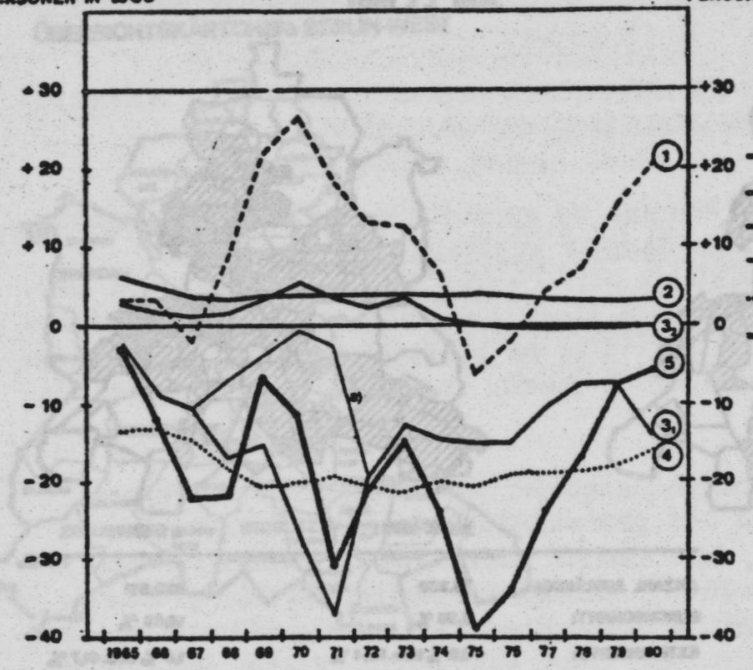

wavoenumassalloen mir.

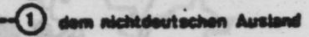

(2) der DOR und Eerlin-Oat

(3) dem Eundeegeblet, Deutsche

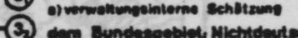

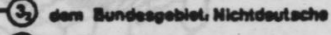

(4) GEBuRtendefizt msgesuat

(3) sevólKeruncsazmaHme msossum

- Maresdurchschertte sel.

(1) +2.480

(2) +3.670

(3) -1.740

Arreonen

a) - 4.90

(3) +1.570

(4) - 10.550

(5) 10.000

Abas

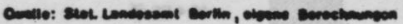

DIE ALTERSSTRUKTUR DER FORTGESCHRIEBENEN WOHNBEVÖLKERUNG VON BERLIN-WEST AM LLßS: (FÖNFJAHRESALTERSKLASSEN)

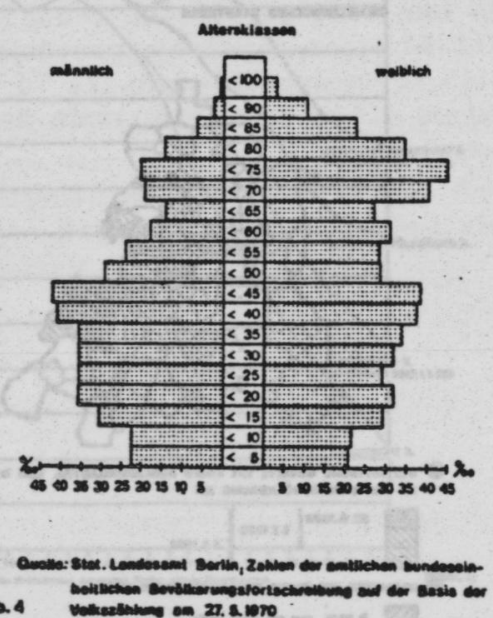

ALTERSSPEZIFISCHE FRUCHTBARKEIT DER FRAUEN IN BERLIN-W - 1980

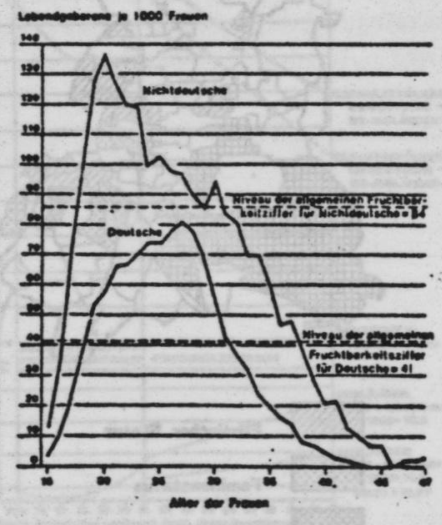

